\title{
DISLOCATION OF THE FIFTH GARPO-METACARPAL JOINT
}

\author{
H. R. Ker, London, England \\ Surgical Registrar, Charing Cross Hospital Group (Wembley Hospital)
}

Isolated dislocation of the carpo-metacarpal joint of the little finger is rare. The fifth carpo-metacarpal joint is less mobile and more protected than the corresponding joint of the thumb, dislocation of which is fairly common, especially in association with Bennett's fracture. A similar injury occurs in the fifth carpo-metacarpal joint, and about twenty cases have been described. There is medial and proximal displacement of the bone, and there may be a fracture of the lateral part of the base, the smaller fragment remaining in its normal position. As in Bennett's fracture-dislocation, the deformity is maintained by muscle pull, in this case the flexor carpi ulnaris acting through the piso-metacarpal ligaments, and continuous traction has been found necessary to secure stable reduction. One such case has been seen recently.

Dislocation with antero-lateral displacement of the metacarpal base across the palmar surface of the neighbouring metacarpals is an injury of much greater rarity. Reference to only four cases has been found, and the present case appears to be the first diagnosed immediately after injury. All have been caused by direct violence to the ulnar side of the hand. In all, closed reduction was found to be impossible, and open reduction has been performed.

McWhorter (1918) described a case in a man of twenty-three. The dislocation was diagnosed two weeks after the injury. After an extensive dissection, reduction was achieved and found to be stable. Good function resulted.

Buzby's case (1934) occurred in a woman of forty-eight from forcibly turning the steering wheel of a car. During open reduction one month later incisions on the palmar and dorsal surfaces of the hand were required, the dislocation was reduced, and the metacarpal base sutured in position with catgut. There was residual weakness of grip.

Roberts and Holland (1936) mentioned a radiograph of such a case, but gave no clinical details.

Watson-Jones (1943) described this injury in a policeman of twenty-eight who had fallen from a motor cycle. Seven weeks after the injury an incision was made over the ulnar border of the hand, and the bone was pulled into place with a blunt hook. The joint surfaces were so eroded that arthrodesis was performed. The hand recovered normal function.

\section{CASE REPORT}

The patient was a joiner of sixty-nine. Both his hands were already stiff from arthritis, and he had had a severe infection in the right hand in his youth, with the result that this was the stiffer hand. When crossing the road he was struck from behind on the ulnar border of his right hand by a motor cycle. On admission to Wembley Hospital he complained of severe pain along the ulnar side of the hand. In spite of the displacement (Fig. 1) the hand differed surprisingly little from the normal. There was a half-inch long laceration on the dorsal surface, and the whole hand was swollen. This swelling, together with extreme tenderness, masked the presence of the metacarpal base in the palm, and its absence from the hypothenar eminence. With the fingers held in semi-flexion, the little finger appeared merely to be abducted slightly. This appearance probably accounts for the delayed diagnosis in the previous cases. When, however, the finger was flexed, it was found to point not at the tubercle of the scaphoid, as is usual, but towards the distal end of the metacarpal bone of the thumb. This appearance of the flexed finger was most striking. Function of the ulnar nerve was normal. 
Radiographs showed a dislocation of the carpo-metacarpal joint, with the base of the metacarpal displaced anteriorly and laterally, lying in front of the base of the third metacarpal. There were two tiny flakes of bone apparently avulsed from the medial side of the base.

Manipulative reduction failed. Preparation was therefore made for open reduction. Operation - Two days after the injury an incision was made along the ulnar border of the hand, the hypothenar muscles were displaced forwards, and an attempt was made to pull the bone back into place with a blunt hook. This proved difficult, and it was supposed that such a blind manoeuvre might endanger the deep branch of the ulnar nerve. The small laceration on the dorsum was therefore excised, and this opening enabled the handle of a scalpel to be used to lever the bone into place. The reduction was unstable because all the ligaments attached to the metacarpal base were ruptured. A hole was therefore drilled in the medial part of the base, and a catgut suture was passed through this from the dorsal incision, and brought back via the medial incision through the ligaments on the medial side of the hamate, constructing a new medial ligament. Non-absorbable material was not used

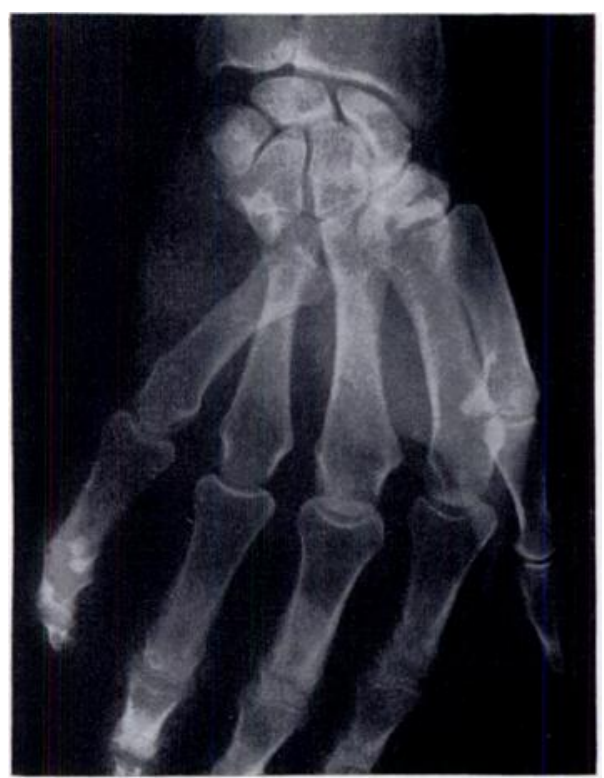

FIG. 1

Initial radiograph.

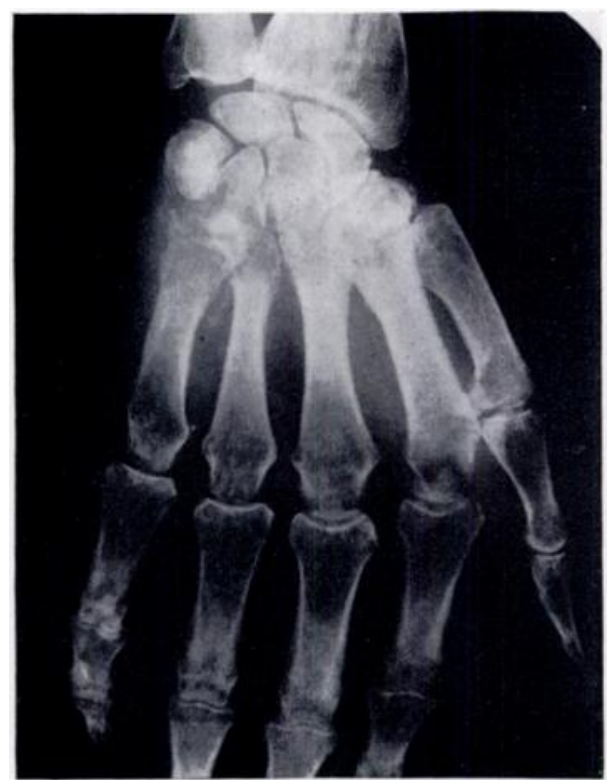

FIG. 2

Six weeks later.

because of the laceration on the dorsum of the hand. Both skin incisions were closed and the hand was immobilised in plaster with the flexed little finger pointing towards the tubercle of the scaphoid.

Progress - Active movements of the other fingers were started immediately. After ten days the sutures were removed, and the little finger was immobilised in semi-flexion. Immobilisation was discontinued after a further four weeks (Fig. 2 ) and active movements of all fingers were started. Recovery of movement was very slow as the whole hand had previously been stiff, and the grip was at first weak. The patient returned to his work as a joiner five months after the injury.

When he was finally discharged a month later the movements of the fingers were still not full, but he stated that they were as good as before the accident. The grip was strong and there was no tenderness over the affected joint. The normal antero-posterior movements of the fourth and fifth carpo-metacarpal joints were full and painless and there was no instability. 


\section{DISCUSSION}

With the exception of the first, there is not a great deal of movement in the carpometacarpal joints. The second and third are virtually immobile, and on them depends the strength of the hand. There is a small amount of antero-posterior movement in the fourth joint, and more in the fifth, so that the fifth metacarpal is capable of moving through about 15 degrees. These are accessory movements which are important for the function of the hand. They bring about the normal cupping of the palm when grasping a rounded object, and assist in opposition of the little finger. Flexion of the fourth and fifth metacarpals occurs also when the fist is clenched in firm gripping. Arthritis in these joints therefore interferes with the mobility of the hand, and weakens the grip. This was the main complaint of the present patient before recovery was complete, and was the residual disability in Buzby's case.

It seems probable that the correct treatment for such a dislocation is early open reduction, with reconstruction of the ligaments. If reduction is undertaken some time after the injury when the joint surfaces are damaged, it should be followed by arthrodesis, as described by Watson-Jones, in order to prevent persistent pain and weakness.

\section{SUMMARY}

Descriptions of four previous cases of antero-lateral dislocation of the fifth carpometacarpal joint are reviewed and a further case is reported. Suggestions are made on treatment.

I wish to thank Mr A. J. Cokkinis for permission to report this case, and Mr David Trevor for his help and advice in the preparation of the paper.

\section{REFERENCES}

BuzBy, B. F. (1934): Palmar Carpo-Metacarpal Dislocation of the Fifth Metacarpal. Annals of Surgery, $100,555$.

McWhorter, G. L. (1918): Isolated and Complete Dislocation of the Fifth Carpometacarpal Joint: Open Operation. Surgical Clinics of Chicago, ii, 793.

Roberts, N., and Holland, C. Thurstan (1936): Isolated Dislocation of the Base of the Fifth Metacarpal. British Journal of Surgery, 23, 567.

Watson-Jones, R. (1943): Fractures and Joint Injuries. Third edition. Edinburgh: E. \& S. Livingstone. 